\title{
Time-Dependent and Force-Dependent Vasoreactivity of Isolated Human Umbilical Arteries
}

\author{
Đorđe Đukanović, ${ }^{1}$ Milica Gajić, ${ }^{1}$ Ranko Škrbić ${ }^{1}$
}

\begin{abstract}
Background/Aim: There have been different experimental conditions for in vitro studies on human umbilical arteries (HUA) in tissue bath system. This diversity was mainly reflected in variables such as stretching tension, incubation period and initial constriction challenging with potassium $(\mathrm{KCl})$. The aim of the study was to establish optimal experimental conditions which will provide better responsiveness of HUA preparations, as well as to examine the impact of $24 \mathrm{~h}$ cold storage on viability and responsiveness of $\mathrm{HUA}$ to $\mathrm{KCl}$ and serotonin.

Methods: The KCl-induced constrictions at different stretching tensions $(0.5 \mathrm{~g}, 1.0$ g, $2.0 \mathrm{~g}$, $4.0 \mathrm{~g}$ ), incubation times ( $30 \mathrm{~min}, 60 \mathrm{~min}, 120 \mathrm{~min}$ ), and after multiple initial constriction challenging were compared. Dose response curves for serotonin were obtained under different conditions (1.0 g and $60 \mathrm{~min}$ vs. $2.0 \mathrm{~g}$ and $120 \mathrm{~min}$ ). The influence of $24 \mathrm{~h}$ cold storage on KCl- and serotonin- induced vasoconstriction of HUA preparations was examined as well.

Results: The strongest constrictions induced by serotonin or $\mathrm{KCl}$ were obtained when preparations were adjusted at $2.0 \mathrm{~g}$ and incubated for $120 \mathrm{~min}$. The KCl-induced constrictions observed after 120 min were statistically higher $(p<0.05)$ when preparations were challenged three times (30 min, $60 \mathrm{~min}, 120 \mathrm{~min}$ ), compared to those challenged only once. The preparations that were stored at $4{ }^{0} \mathrm{C}$ for $24 \mathrm{~h}$ showed significantly stronger serotonin-induced constrictions $(\mathrm{p}<0.01)$. The cold storage had no influence on KCl-induced constriction.

Conclusion: For performing in vitro studies on HUA preparations in tissue bath, we propose stretching tension of $2.0 \mathrm{~g}$, incubation period of $120 \mathrm{~min}$ and multiple initial constriction challenging with $\mathrm{KCl}$ as optimal experimental condition. We also showed that HUA preparations retained functional viability even after $24 \mathrm{~h}$ of cold storage.
\end{abstract}

Key words: Human umbilical artery; Stretching tension; Incubation time; Constriction; Serotonin.
(1) Centre for Biomedical Research, Faculty of Medicine, University of Banja Luka, Banja Luka, the Republic of Srpska, Bosnia and Herzegovina.

Correspondence:

ĐORĐE ĐUKANOVIĆ

E: djordje.djukanovic@med.unibl.org

\section{ARTICLE INFO}

Received: 25 August 2020 Accepted: 11 September 2020

\section{Introduction}

In addition to numerous animal models of pregnancy and sporadic human clinical trials, studies on isolated umbilical blood vessels have a special place in research related to pregnancy complications. The in vitro studies on umbilical arteries and veins allow direct assessment of vascular reactivity of utero-placental and foeto-placental circulation. ${ }^{1}$ However, studies on animal blood vessel preparations cannot completely replace human preparations and their pathophysiological models. ${ }^{2}$ Therefore, human umbilical blood vessels are of particular importance, since they 
can provide the closest possible image of the in vivo system. The importance of the HUA has long been recognised, and its use in research dates back to the 1950s. ${ }^{3}$ Umbilical cord is one of the few human tissues that is widely available and provides a high yield of HUA preparations.

Majority of the research studies on HUA are focused on elucidating the vascular dysfunction that occurs in preeclampsia, which is associated with an increase in utero-placental vascular resistance. ${ }^{4}$ It is well known that HUA are not innervated blood vessels, ${ }^{5}$ and that their vasoactivity is regulated exclusively by autacoids from blood circulation, such as serotonin, histamine, bradykinin, angiotensin, oxytocin, as well as by different eicosanoids. ${ }^{6}$ Due to the described specificity of umbilical circulation, it is assumed that these vasoactive agents may play an important role in the pathogenesis of preeclampsia.

Since it was found that mechanical properties and reactivity of umbilical blood vessels are changed in preeclampsia, ${ }^{6,7}$ it is important to establish optimal experimental conditions for physiological models that will allow for the interpretation of these changes. The length and stretch of HUA preparations, the duration of incubation time, the composition of the solution to maintain the viability of the preparations and the initial constriction challenging procedures are some of the key determinants in defining the conditions that must be achieved before performing experiments in the tissue bath system. The majority of authors apply the same conditions regarding the form and length of the HUA preparation (rings, 3-4 cm long), aeration of the HUA preparation (mixture of $95 \% \mathrm{O}_{2}$ and $5 \% \mathrm{CO}_{2}$ ) and composition of the nutrient solution (Krebs-Ringer bicarbonate solution), ${ }^{6-10}$ whereas the differences are reflected mainly in passive tension, incubation period and initial constriction challenging. Given the existence of great diversity in the application of physical properties for testing HUA vasoactivity, it is of particular importance to establish an optimisation of the tissue bath methodology that will allow better responsiveness of HUA preparations.

The aim of the study was to analyse the effects of different physical variables such as stretching tension, incubation time, initial constriction challenging and cold storage period to determine the optimal conditions for measuring the vasoactivity of isolated HUA.

\section{Methods}

\section{Ethical principles}

The present study was conducted with the approval of the Ethics Committee for Research on Humans and Biological Materials, at the Faculty of Medicine, University of Banja Luka, the Republic of Srpska, Bosnia \& Herzegovina (B\&H).

\section{Tissue preparation}

Umbilical cords were obtained from healthy pregnant women after full-term vaginal delivery or Caesarean section at the Clinics for Gynaecology and Obstetrics, University Clinical Centre of the Republic of Srpska (Banja Luka, B\&H). After delivery, the segments of umbilical cords (5-10 $\mathrm{cm}$ in length) were immediately placed into the modified Krebs-Ringer bicarbonate solution and transported on the ice to the Laboratory of Pharmacology and Toxicology, Centre for Biomedical Research, Faculty of Medicine, University of Banja Luka. For this purpose, the modified Krebs-Ringer solution with less calcium ions than standard solution $\left(\mathrm{CaCl}_{2} 0.16 \mathrm{mmol} / \mathrm{L}\right)$ was used.

The umbilical artery was cleaned of Wharton jell and the connective tissue was carefully removed. The cleaning was performed in Petri dishes filled with modified Krebs-Ringer solution on the flat ice pack. The preparation was cut into rings of 3-4 $\mathrm{mm}$ in length and each ring was suspended between two stainless steel wires in a jacketed tissue bath containing $20 \mathrm{~mL}$ Krebs-Ringer bicarbonate solution $\left(37^{\circ} \mathrm{C}, \mathrm{pH} 7.4\right)$, aerated with a mixture of $95 \% \mathrm{O}_{2}$ and $5 \% \mathrm{CO}_{2}$.

One of the wire hooks was attached to a transducer (CH1-SN: IT) connected to the amplifier (SN:BS 007) with recording system (Fast Acquisition, Elunit Group, Serbia) that recorded changes in isometric tension, using an e-Lab software. The second wire hook was attached to a displacement unit allowing fine adjustments of passive tension (g). During equilibration time, the organ bath solution was changed every 10 minutes and tension was adjusted when necessary.

\section{Experimental protocols}

Resting tension and incubation time

In order to evaluate the optimal resting tension and incubation period for HUA preparations, the different stretching conditions and incubation times were performed to obtain maximal con- 
striction induced by potassium chloride $(\mathrm{KCl})$. At the beginning of experiment the rings were equilibrated unstretched in organ bath at $37^{\circ} \mathrm{C}$ for 30 minutes. After that, the preparations were divided in four groups and each preparation was stretched to a different resting tension: $0.5 \mathrm{~g}, 1.0$ $\mathrm{g}, 2.0 \mathrm{~g}$ and $4.0 \mathrm{~g}$. When rings reached the determined tension, $\mathrm{KCl}(40 \mathrm{mM})$ was applied in precisely defined time points: $30 \mathrm{~min}, 60 \mathrm{~min}$ and $120 \mathrm{~min}$. Between each $\mathrm{KCl}$ addition, rings were washed for several times and then adjusted to a determined resting tension.

\section{$\mathrm{KCl}$-induced constriction challenge}

The intention was to assess the influence of the repeated initial constriction with $\mathrm{KCl}$ when preparations were set on optimal resting tension of $2.0 \mathrm{~g}$. After $30 \mathrm{~min}$ of incubation time, the preparations were stretched to a passive force of 2.0 g. One group of preparations were allowed to equilibrate for $120 \mathrm{~min}$ and then were exposed to $\mathrm{KCl}$ initial challenge $(40 \mathrm{mM})$, while on the other group of preparations three initial $\mathrm{KCl}$ challenges $(40 \mathrm{mM})$ were performed, at $30 \mathrm{~min}, 60 \mathrm{~min}$ and $120 \mathrm{~min}$, as previously described.

\section{Serotonin dose-response curve}

The aim of this protocol was to compare the dose-response curves for serotonin in HUA preparations under different passive tension and incubation time variables. When the first incubation period of $30 \mathrm{~min}$ ended, the preparations were stretched and equilibrated at different conditions. One group of preparations was stretched at $1.0 \mathrm{~g}$ and incubated for $60 \mathrm{~min}$, while the other group was stretched at $2.0 \mathrm{~g}$ and incubated for $120 \mathrm{~min}$. All preparations were exposed to $\mathrm{KCl}$ $(40 \mathrm{mM})$ in order to obtain a reference constriction (100\%). After several wash-out periods, when preparations achieved the stable resting tension, a cumulative concentration-response curve for serotonin $\left(10^{-8}-3 \times 10^{-5} \mathrm{M}\right)$ was obtained. At the end of each experiment, the viability of preparation was tested by challenging it with KCl-induced constriction.

\section{Cold storage}

Within this protocol the intention was to examine the effect of cold storage on HUA vasoreactivity. Optimal conditions (incubation period of $2 \mathrm{~h}$ and passive tension of $2.0 \mathrm{~g}$ ) established in previous protocols were applied at HUA preparations after $24 \mathrm{~h}$ of cold storage at $4{ }^{\circ} \mathrm{C}$. At the beginning of experiment, $\mathrm{KCl}$-induced constrictions $(40 \mathrm{mM})$ were established and after several washing-out periods, when basal line was stable, a cumulative concentration response curves for serotonin $\left(10^{-8}\right.$ $-3 \times 10^{-5} \mathrm{M}$ ) were obtained.

\section{Drugs and solutions}

A Krebs-Ringer bicarbonate solution of the following composition was used (mmol/L): $\mathrm{NaCl} 118.3$, $\mathrm{KCl}$ 4.7, $\mathrm{CaCl}_{2} 2.5, \mathrm{MgSO}_{4} 1.2, \mathrm{NaHCO}_{3} 25, \mathrm{KH}_{2} \mathrm{PO}_{4}$ 1.2, and glucose 5.6. All stock solutions and serial dilutions were made in distilled water and were prepared shortly before the start of each of the experiments. Serotonin was obtained from Sigma Chemical Co. (St. Louis, MO, USA) and potassium chloride $(\mathrm{KCl})$ was obtained from Lach: Ner (Zagreb, Croatia).

\section{Statistical analysis}

Results were presented as mean \pm standard error (mean \pm SEM) for number of preparations studied. Figures and statistical analysis were performed using SigmaPlot 14.0 (Systat Software Inc.). Two-way ANOVA with repeated measures was used to compare concentration-response curves for serotonin. Statistical significance between two groups was determined by Student's $t$-test and the $p$ values less than 0.05 were considered to be significant.

\section{Results}

\section{Resting tension and incubation time}

Preparations of HUA showed different responses to $\mathrm{KCl}$ at various stretching tensions and incubation periods. Preparations that were stretched at $0.5 \mathrm{~g}$ and incubated for $30 \mathrm{~min}$ did not show any response to $\mathrm{KCl}$, while other preparations that were stretched at tensions of 1.0, 2.0, $4.0 \mathrm{~g}$ showed very weak constrictions. By prolonging the incubation time to 60 or $120 \mathrm{~min}$, all preparations showed stronger constrictions than those obtained at $30 \mathrm{~min}$. When preparations were stretched at tension of 2.0 and $4.0 \mathrm{~g}$ the constrictions were stronger than those stretched at $0.5 \mathrm{~g}$, but the significance was confirmed only for $2.0 \mathrm{~g}$. Further prolongation of incubation time to 120 min resulted in significant increase in constrictions for preparations stretched at tension of 2.0 and $4.0 \mathrm{~g}$. However, additional increase in stretching tension of $4.0 \mathrm{~g}$ was not followed by stronger constriction response, as it would be expected (Figure 1). 


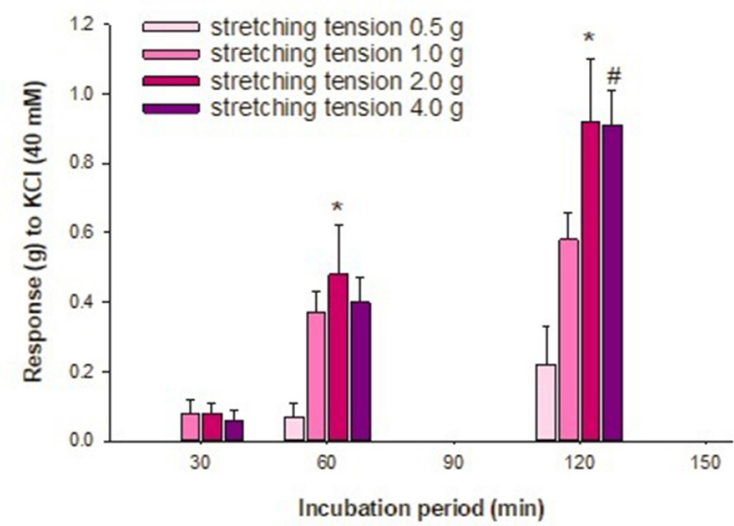

Figure 1: Comparison of KCl-induced constrictions, at a different stretching tensions and incubation periods, in human umbilical arteries in vitro. Preparations were adjusted at: $0.5 \mathrm{~g}(n=6), 1.0 \mathrm{~g}(n=8), 2.0 \mathrm{~g}(n=10)$ or $4.0 \mathrm{~g}(n=6)$ and each group was incubated for $30 \mathrm{~min}, 60 \mathrm{~min}$ and 120 min. Results (mean \pm SEM) are expressed in grams of KCl-induced constriction.

${ }^{*} p<0.05 ; \# p<0.05$ (2.0 g vs. 0.5 g; 4.0 g vs. 0.5 g, respectively).

\section{Potassium-induced constriction challenge}

$\mathrm{KCl}$-induced vasoconstriction $(40 \mathrm{mM})$ was significantly stronger in HUA preparations that were exposed to three initial challenges (separate, non-cumulative additions of $\mathrm{KCl}$ at $30 \mathrm{~min}$, $60 \mathrm{~min}$ and $120 \mathrm{~min}$ ), compared to the preparations that were exposed to only one initial challenge (at $120 \mathrm{~min}$ ) (Figure 2).

\section{Serotonin dose-response curve}

Concentration-response curve for serotonin, with the stretching tension of $2.0 \mathrm{~g}$ and incubation pe-

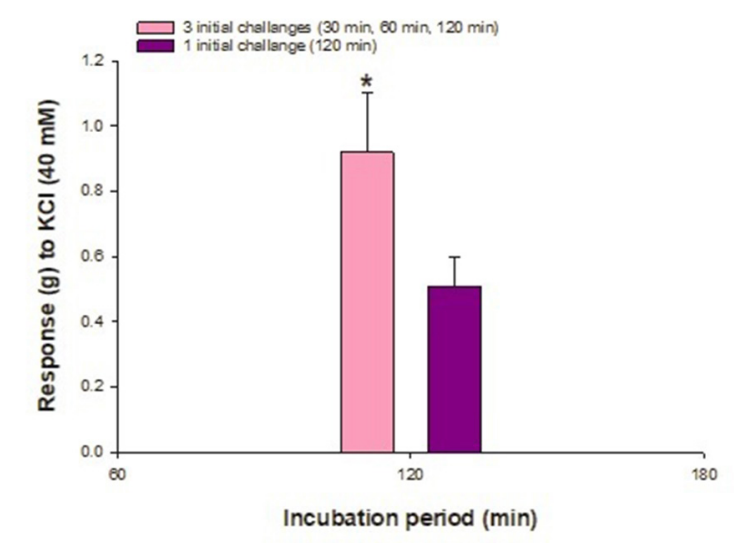

Figure 2: Comparison of KCl-induced constrictions of human umbilical arteries in vitro, in the absence or presence of first two initial challenges with $\mathrm{KCl}$, at the optimal resting tension of $2 \mathrm{~g}$. One group of preparations $(n=10)$ was exposed to three initial challenges: $30 \mathrm{~min}, 60 \mathrm{~min}$ and 120 min (pink bar), while the other group of preparations $(n=6)$ was exposed to one initial challenge: 120 min (purple bar). Results (mean \pm SEM) are expressed in grams of $\mathrm{KCl}$-induced constriction.

${ }^{*} p<0.05$ (significantly different from the respective control).

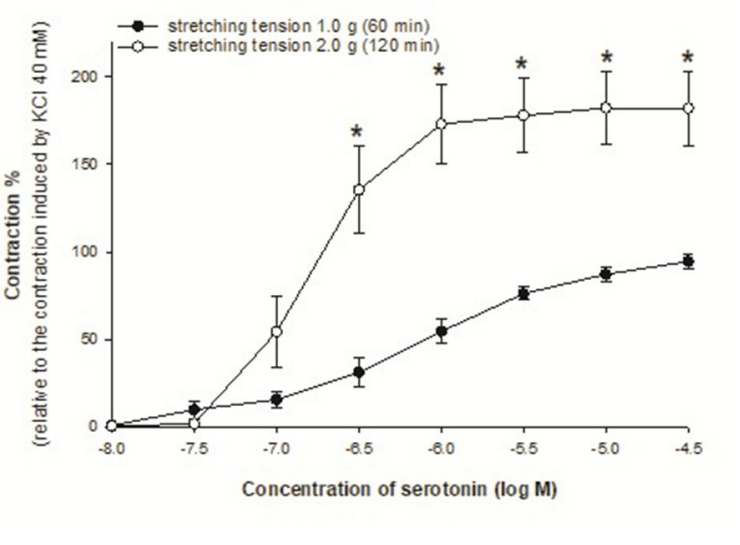

Figure 3: Comparison of concentration-response curves for serotonin, at different stretching tension and incubation time of human umbilical arteries preparations in vitro. Results (mean \pm SEM) are expressed in percentage of reference $\mathrm{KCl}$-induced constriction.

${ }^{*} p<0.001$ (significantly different from the respective control).

riod of $120 \mathrm{~min}$, showed a significantly stronger $(p<0.001)$ constrictions compared to the concentration-response curve at stretching tension of $1.0 \mathrm{~g}$ and incubation period of $60 \mathrm{~min}$ (Figure 3).

\section{Cold storage}

Preparations that were stored at the temperature of $4{ }^{\circ} \mathrm{C}$ for $24 \mathrm{~h}$ did not show any significant difference in the magnitude of the $\mathrm{KCl}$-induced constriction compared to the preparations of HUA that were used as fresh preparations, without cold storage (Figure 4).

Serotonin-induced constrictions were significantly stronger $(p<0.01)$ in cold-stored HUA preparations. Concentration-response curves for serotonin indicated higher efficacy of serotonin

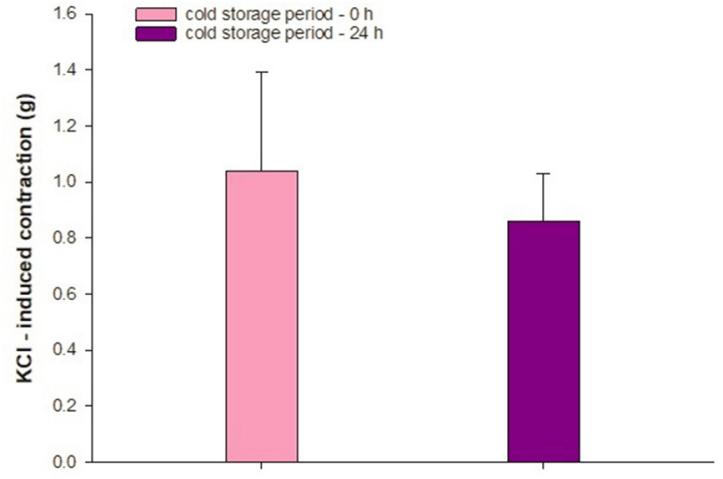

Figure 4: Comparison of $\mathrm{KCl}$-induced constrictions of human umbilical arteries in vitro, at different cold storage times of human umbilical arteries preparations. Results (mean \pm SEM) are expressed in grams of KCl-induced constriction 


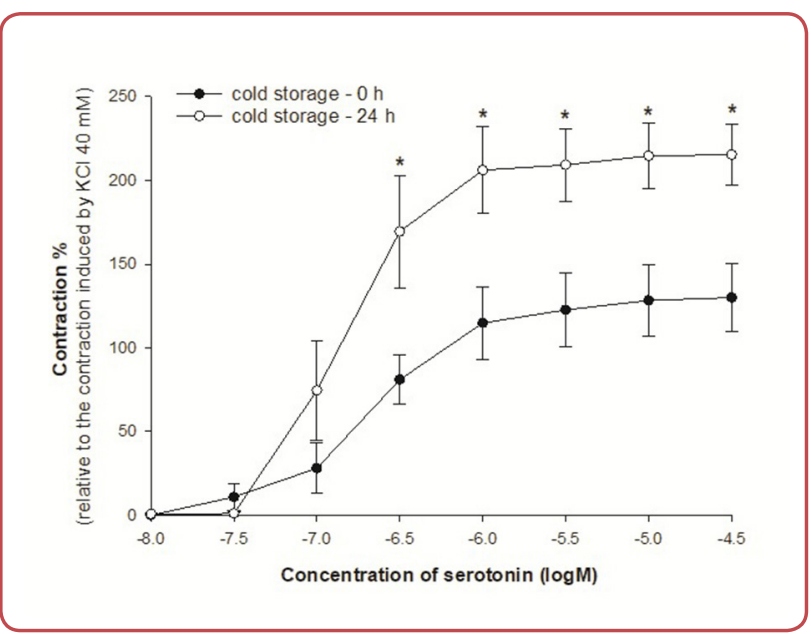

Figure 5: Comparison of concentration-response curves for serotonin, at different cold storage times of human umbilical arteries preparations in vitro. Results (mean $\pm S E M$ ) are expressed in percentage of reference $\mathrm{KCl}$-induced constriction.

${ }^{*} p<0.01$ (significantly different from the respective control).

in cold-stored preparations, since significantly higher magnitude of responses were found at higher concentrations of serotonin $\left(3 \times 10^{-7}-10^{-5}\right.$ M). However, at lower concentrations $\left(10^{-8}-10^{-7}\right.$ M) there were no significance in the magnitude of the response, indicating that cold storage of the HUA preparation did not affect serotonin sensitivity (Figure 5).

\section{Discussion}

The main purpose of this study was to determine the optimal stretching tension and incubation period for researches that use HUA in tissue bath, as well as to examine the effect of multiple initial challenging and cold storage on HUA vasoactivity.

Bertrand et al reported that for reliable measurement of pharmacological properties it is necessary to stretch the HUA preparations to 5.0 or $6.0 \mathrm{~g}$, while other investigators in previous studies established the resting tension of $2.0 \mathrm{~g}$ as an optimal tension for HUA preparations. ${ }^{8,9}$ In addition, there are reports that applied a stretching tension of $4.0 \mathrm{~g}$ is optimal during $4 \mathrm{~h}$ of incubation, ${ }^{10,11} \mathrm{al}-$ though in most studies the incubation time was 2 h. $^{8,9,12}$

Results of these experiments have shown that the response of HUA to $\mathrm{KCl}$ was stronger after increasing stretching tension and incubating period. The maximal response was obtained when preparations were stretched at $2.0 \mathrm{~g}$ and incu- bated for $120 \mathrm{~min}$. Despite the fact that after 120 min there was no significant difference between responses of arteries stretched at $2.0 \mathrm{~g}$ and 4.0 $\mathrm{g}$, the stretching tension of $4.0 \mathrm{~g}$ was harder to achieve, thus, $2.0 \mathrm{~g}$ and $120 \mathrm{~min}$ are suggested as optimal experimental conditions. The sensitivity of HUA to $\mathrm{KCl}$ was not affected by stretching tension since no significant difference in responses was found between the HUA preparations stretched at 1.0, 2.0 or $4.0 \mathrm{~g}$, although the differences in constrictions were significant when the mentioned tensions were compared with the lowest one applied, $0.5 \mathrm{~g}$. This is in accordance with the intact sensitivity of chorionic vessels to KCl.1 However, in HUA preparations stretched at minimal passive tension $(0.5 \mathrm{~g})$, the incubation time might have an effect on the sensitivity to $\mathrm{KCl}$, since constrictions were absent after $30 \mathrm{~min}$ of incubation, while prolongation of incubation time enhanced responsiveness.

In order to assess the functional integrity of HUA preparations, most authors use $\mathrm{KCl}$ as a reference contraction activator, applying different protocols to achieve initial challenge. The concentration of $\mathrm{KCl}$ that caused the maximal constriction for HUA preparations was found to be $80 \mathrm{mM}$, while the submaximal concentrations ranged from 20 $\mathrm{mM}$ to $60 \mathrm{mM} .{ }^{9}$ However, Bertrand et al applied initial challenge with $100 \mathrm{mM}$ of $\mathrm{KCl}$, usually with repetitions of 3 to 4 times, ${ }^{6}$ while Tufan et al used $40 \mathrm{mM} \mathrm{KCl}$ for contractile response and repeated it twice, the second constriction of which was taken as a reference one. ${ }^{10}$ Estañ et al asserted that $40 \mathrm{mM} \mathrm{KCl}$ was the optimal concentration that would reach the submaximal constriction in foeto-placental blood vessels. ${ }^{1}$ Considering the all above mentioned findings, it was decided to use $\mathrm{KCl}$ as a reference contractile agent at a concentration of $40 \mathrm{mM}$. Our results have shown that greater constrictions were obtained after multiple challenging with $40 \mathrm{mM} \mathrm{KCl}$. The assumption for presence of greater constrictions and thereby increase of intracellular calcium ions $\left(\mathrm{Ca}^{2+}\right)$ could be that repeated $\mathrm{KCl}$-induced membrane depolarisation lead to prolonged activation of $\mathrm{Ca}^{2+}$ voltage channels and release from intracellular depots..$^{13}$ It is also possible that during multiple challenging a higher amount of $\mathrm{Ca}^{2+}$ remains in the cell which is caused by inappropriate $\mathrm{Ca}^{2+}$ displacement after every wash-out period. ${ }^{14}$

It has long been known that serotonin is one of the most potent vasoconstrictors of HUA. ${ }^{8}$ Finding that concentration of serotonin in umbilical 
vessels at birth was $10^{-7} \mathrm{M}^{15}$ determined the range of serotonin concentrations most commonly used for in vitro studies. ${ }^{6,11,16}$ In the present study the dose-response curves for serotonin $\left(10^{-8}-3 \mathrm{x}\right.$ $10^{-5} \mathrm{M}$ ), obtained after different stretching tensions and incubation periods, showed force- and time-dependent constrictions. It was found that the efficacy of serotonin was significantly higher under the optimal experimental conditions established in this study (2.0 g and $120 \mathrm{~min}$ ). However, sensitivity to serotonin was not affected when preparations were stretched either at $1.0 \mathrm{~g}$ for 60 $\mathrm{min}$ or at $2.0 \mathrm{~g}$ for $120 \mathrm{~min}$. It has been previously reported by Tufan et al that maximal serotonin constriction for HUA, expressed as percentage of KCl-induced constriction, was approximately 130 $\%{ }^{10}$ Dayigolu et al applied the same experimental conditions ( $4.0 \mathrm{~g}$ and $4 \mathrm{~h}$ ) and achieved a maximal constriction of approximately $145 \%{ }^{11}$ In the present experiment, higher maximal constriction for serotonin (approximately $180 \%$ ) were reached, using lower stretching tension $(2.0 \mathrm{~g})$ and shorter incubation time (120 min). Therefore, the advantages of this approach are reflected on savings of time and efforts in settings of HUA preparations for experiments.

In HUA preparations that were exposed to $24 \mathrm{~h}$ cold storage at $4{ }^{\circ} \mathrm{C}$, the $\mathrm{KCl}$ produced qualitatively less constrictions compared to those in non-cold storage HUA preparations, but this difference was not statistically significant. Nevertheless, Sinanović and Chiba reported that KCl-induced constrictions were significantly decreased in dog and monkey skeletal muscle arteries after 3-5 days storage at $4{ }^{\circ} \mathrm{C} .{ }^{17,18}$ They suggested that hypothermic conditions might have led to the change in sensitivity of $\mathrm{Ca}^{2+}$ voltage channels and thus $\mathrm{Ca}^{2+}$ influx. Moreover, it has been shown that cold storage $\left(24-72 \mathrm{~h}\right.$ at $\left.4{ }^{\circ} \mathrm{C}\right)$ of rat thoracic aorta also diminished $\mathrm{KCl}$-induced constrictions and that reactive oxygen species may be responsible for storage-induced changes in vascular reactivity. $^{19}$

On the other hand, serotonin showed significantly higher efficacy, but not sensitivity in HUA preparations that were exposed to $24 \mathrm{~h}$ cold storage, compared to the control HUA preparations. Although the percentage of achieved serotonin constriction was almost twice as high in the preparations exposed to cooling, it still does not represent a realistic image of the increase in serotonin efficacy, taking into account that the refer- ence $\mathrm{KCl}$ constrictions were lower in these preparations. The effects of cold storage on serotonin vasoactivity have been investigated in various blood vessel preparations, but the results were not consistent. There are reports that show that cold storage did not modify vascular response of serotonin on dog and monkey muscle arteries, ${ }^{17,18}$ whereas other reported that serotonin-induced constriction of rat aorta was almost completely diminished after $72 \mathrm{~h}$ of cooling at $4{ }^{\circ} \mathrm{C} .{ }^{19}$ Interestingly, Kevelaitis et al. found that endothelial dysfunction during cold preservation enhanced the sensitivity of serotonin on rat coronary arteries. ${ }^{20}$ Since the results of the present experiments showed that cold storage increased serotonin constrictions, further studies are needed in order to examine the role of endothelial dysfunction on this phenomenon.

Like in the optimisations already performed for isolated human pulmonary arteries, ${ }^{2}$ as well as for isolated human chorionic arteries, ${ }^{1,21}$ the results presented here represent an optimisation of the methodology for isolated HUA.

\section{Conclusion}

Results from the present study indicate that both $\mathrm{KCl}$ - and serotonin-induced constrictions were higher when HUA preparations were adjusted at $2.0 \mathrm{~g}$ stretching force and $120 \mathrm{~min}$ incubation period and that multiple initial challenging enhance vascular response to $\mathrm{KCl}$. It was also shown that HUA preparations preserved functional sensitivity during $24 \mathrm{~h}$ cold storage for both $\mathrm{KCl}$ - and serotonin-induced constrictions. Considering all the above, mentioned experimental conditions are proposed as optimal to preform studies on HUA in isolated tissue bath, even $24 \mathrm{~h}$ after sampling.

\section{Acknowledgements}

None.

\section{Conflict of interest}

None. 


\section{References}

1. Estañ L, Abad A, Morales-Olivas FJ, Serra-Serra V. Isolated human chorionic vascular reactivity: technical considerations for fresh preparations. Gen Pharmacol 1998;30(1):89-93.

2. Hussain A, Bennett RT, Chaudhry MA, Qadri SS, Cowen $\mathrm{M}$, Morice $\mathrm{AH}$, et al. Characterization of optimal resting tension in human pulmonary arteries. World J Cardiol 2016;8(9):553-8.

3. Rodegra H, Roder G, Schmermund HJ, Soering K. Wirkung von 5-Hydroxytryptamin auf die isolierte Nabelschnurarterie [Effect of 5-hydroxytryptamine on the isolated umbilical cord artery]. Naunyn Schmiedebergs Arch Exp Pathol Pharmakol 1957;232(1):285-6. German.

4. Tong W, Giussani DA. Preeclampsia link to gestational hypoxia. J Dev Orig Health Dis 2019;10(3):322-33.

5. Reilly FD, Russell PT. Neurohistochemical evidence supporting an absence of adrenergic and cholinergic innervation in the human placenta and umbilical cord. Anat Rec 1977;188(3):277-86.

6. Bertrand C, St-Louis J. Reactivities to serotonin and histamine in umbilical and placental vessels during the third trimester after normotensive pregnancies and pregnancies complicated by preeclampsia. Am J Obstet Gynecol 1999;180(3 Pt 1):650-9.

7. Bertrand C, Duperron L, St-Louis J. Umbilical and placental vessels: modifications of their mechanical properties in preeclampsia. Am J Obstet Gynecol 1993;168(5):1537-46.

8. Altura BM, Malaviya D, Reich CF, Orkin LR. Effects of vasoactive agents on isolated human umbilical arteries and veins. Am J Physiol 1972;222(2):345-55.

9. Monuszko E, Halevy S, Freese K, Liu-Barnett M, Altura B. Vasoactive actions of local anaesthetics on human isolated umbilical veins and arteries. Br J Pharmacol 1989;97(2):319-28.

10. Tufan H, Ayan-Polat B, Tecder-Unal M, Polat G, Kayhan $\mathrm{Z}$, Oğüş E. Contractile responses of the human umbilical artery to $\mathrm{KCl}$ and serotonin in Ca-free medium and the effects of levcromakalim. Life Sci 2003;72(12):1321-9.

11. Dayioglu E, Buharalioglu CK, Saracoglu F, Akar F. The effects of bumetanide on human umbilical artery contractions. Reprod Sci 2007;14(3):246-52.
12. Templeton AG, McGrath JC, Whittle MJ. The role of endogenous thromboxane in contractions to U46619, oxygen, 5-HT and 5-CT in the human isolated umbilical artery. Br J Pharmacol 1991;103(1):1079-84.

13. Kleszczewski T, Buzun L, Lisowska A, Modzelewska B. Potassium induced contraction of the internal thoracic artery in vitro is time related: the potential consequences in the analysis of the mechanism of the spasm after coronary artery bypass grafting and in the analysis of the results of in vitro studies. Heart Vessels 2016;31(4):61621.

14. Wright G. Use-dependent decline in rat aorta sensitivity to contraction by potassium. Can J Physiol Pharmacol 1991;69(7):921-8.

15. Jones JB, Rowsell A. Fetal 5-hydroxytryptamine levels in late pregnancy. J Obstet Gynaecol Br Commonw 1973;80(8):687-9.

16. Okatani Y, Wakatsuki A, Reiter RJ. Melatonin suppresses homocysteine enhancement of serotonin-induced vasoconstriction in the human umbilical artery. J Pineal Res 2001;31(3):242-7.

17. Sinanović $\mathrm{O}$, Chiba S. Responsiveness of skeletal muscle branches of the dog femoral artery to alpha-adrenoceptor agonists before and after cold storage. Arch Int Pharmacodyn Ther 1987;287(1):146-57.

18. (Sinanović O, Chiba S. Responsiveness of monkey skeletal muscle arteries to vasoconstrictor substances before and after cold storage. Jpn J Pharmacol 1988;46(3):23746.

19. Güney HZ, Ercan ZS. Prolonged cold storage diminishes the 5-hydroxytryptamine- and potassium chloride-mediated contractions of rat thoracic aorta. Tohoku J Exp Med 2009;217(2):155-61.

20. Kevelaitis E, Nyborg NC, Menasché P. Coronary endothelial dysfunction of isolated hearts subjected to prolonged cold storage: patterns and contributing factors. J Heart Lung Transplant 1999;18(3):239-47.

21. Abad A, Estañ L, Morales-Olivas FJ, Serra V. Reactivity of isolated human chorionic vessels: analysis of some influencing variables. Can J Physiol Pharmacol 2003;81(12):1147-51. 